\title{
Dibenzoazepine analogues: the electrophysiological properties of JL3, a potential atypical antidepressant
}

\author{
Jean-François Liégeois ${ }^{a, b, *}$, Jacqueline Scuvée-Moreau ${ }^{c}$, Irène Giesbers ${ }^{c}$, Jacques Damas ${ }^{b}$, \\ Jacques Bruhwyler ${ }^{\mathrm{d}}$, Joseph Géczy ${ }^{\mathrm{d}}$, Jacques Delarge ${ }^{\mathrm{a}}$, Albert Dresse ${ }^{\mathrm{c}}$ \\ "Laboratory of Medicinal Chemistry, University of Liège, Liège, Belgium \\ b Laboratory of Physiology, University of Liège, Liège, Belgium \\ c Laboratory of Pharmacology, University of Liège, Liège, Belgium \\ 'Therabel Research, Brussels, Belgium
}

Received 4 January 1996; revised 25 April 1996; accepted 7 May 1996

\begin{abstract}
JL3, 10-(4-methylpiperazin-1-yl)pyrido[4,3-b][1,4]benzothiazepine, has potent antidepressant-like activity in Porsolt's test in mice. Therefore, its influence on the electrical activity of central monoaminergic neurons was investigated in rats anaesthetized with chloral hydrate. JL3 induced a marked decrease of the firing rate of dorsal raphe serotonergic neurons $\left(\mathrm{ID}_{50}=3.87 \pm 0.57 \mathrm{mg} \mathrm{kg}^{-1}\right)$ and of locus coeruleus noradrenergic neurons $\left(\mathrm{ID}_{50}=2.63 \pm 0.35 \mathrm{mg} \mathrm{kg}{ }^{-1}\right.$ ). The drug did not modify the electrical activity of A10 dopaminergic neurons. JL3 does not block amine uptake but it has affinity for $5-\mathrm{HT}_{1 \mathrm{~A}}$ and $5-\mathrm{HT}_{2}$ receptors. It is speculated that serotonergic mechanisms could play a role in the electrophysiological effects of JL3.
\end{abstract}

Keywords: JL3 (10-(4-methylpiperazin-1-yl)pyrido[4,3-b][1,4]benzothiazepine); Antidepressant; Dorsal raphe; Locus ceruleus; A10 dopamine neuron; Electrophysiology

\section{Introduction}

Clozapine exhibits antipsychotic activity associated with a beneficial effect on negative symptoms of schizophrenia but combined with various side-effects, such as hypersalivation, seizures or agranulocytosis. In a search for safer clozapine-like compounds, we have developed various pyridobenzazepine derivatives (Liégeois et al., 1993, 1994), some of which show antipsychotic potential in animal models (Bruhwyler et al., 1992). The original compounds were first screened in an open-field test in rats. In this preliminary study, some pyridine derivatives showed strong disinhibitory activity (Bruhwyler et al., 1995) that could be related to an antidepressant and/or anxiolytic potential. In a subsequent study using Porsolt's test, it also appeared

\footnotetext{
${ }^{*}$ Corresponding author. Laboratory of Medicinal Chemistry and Physiology, University of Liège, rue Fusch 3, 4000 Liège, Belgium. Tel.: +32 41322969 or +3241665936 ; fax: +3241221855 .
}

that some of the derivatives had activities similar to those of antidepressant drugs (Bruhwyler et al., 1995). The most interesting compound in this respect, JL3, 10-(4-methylpiperazin-1-yl)pyrido[4,3-b][1,4]benzothiazepine, was found to be equipotent to imipramine (Bruhwyler et al., 1995).

In view of these results, we decided to further investigate the mechanism of action of JL3, using in vivo electrophysiological techniques. Indeed, previous studies have shown that the acute administration of most antidepressant drugs more or less selectively decreases the firing rate of central monoaminergic neurons (Scuvée-Moreau and Dresse, 1979; Dresse and Scuvée-Moreau, 1984). In the present work, we have studied the influence of JL3 on the electrical activity of dorsal raphe serotonergic neurons, locus coeruleus noradrenergic neurons and A10 dopaminergic neurons. The effect of JL3 was compared with that of various antidepressant drugs, previously examined under the same experimental conditions. 


\section{Materials and methods}

\subsection{Electrode implantation and recording}

Male Wistar rats (200-300 g) were anaesthetized with chloral hydrate $(400 \mathrm{mg} / \mathrm{kg}$ i.p.) and mounted in a stereotaxic apparatus. Their temperature was maintained at 36$37^{\circ} \mathrm{C}$ by means of a heating pad. A small bone flap was removed above the implantation point and the venous sinus was tied and cut. The electrical activity of the neurons was recorded by means of extracellular glass micropipettes filled with $\mathrm{NaCl} 2 \mathrm{M}$ saturated with Fast green. The electrodes were implanted at the following coordinates: DR $0.0-0.5 \mathrm{~mm}$ anterior, $0.0 \mathrm{~mm}$ lateral to lambda, 4.5-5.5 mm ventral to brain surface; LC 1.7-2.2 mm posterior, 1.1-1.3 mm lateral to lambda, 5.5-6.5 mm ventral to brain surface; A10 1.8-2.2 mm anterior, 0.4-0.6 $\mathrm{mm}$ lateral to lambda, $7.5-8.5 \mathrm{~mm}$ ventral to brain surface. Action potentials were passed through an impedance adapter and an amplifier into a Tektronix 2220 oscilloscope. Signals were also passed into an amplitude discriminator and a digital counter. At the end of the experiment, a spot of Fast green was deposited at the tip of the electrode. The animal was perfused with a solution of formaldehyde $4 \%$ and the brain was removed for the histological control of the position of the electrode.

\subsection{Identification of the cells}

DR neurons were characterized by a regular firing rate and a low frequency of discharge of $0.2-2$ spikes/s (Aghajanian et al., 1978). LC neurons were characterized by a stable firing rate of 0.5-5 spikes/s and a typical response to noxious stimuli (Korf et al., 1974). A10 neurons were characterized by bursts of spikes of decreasing amplitude and a frequency of discharge of 1-9 spikes/s (Bunney et al., 1973).

\subsection{Study of drug action}

The electrical activity of the cell was recorded during a control period of a few minutes in order to determine the baseline firing rate. The mean number of spikes $/ 10 \mathrm{~s}$ was calculated every minute. JL3 was perfused into the jugular vein by means of a perfusion pump (flow $6 \mathrm{ml} / \mathrm{h}$ ). The percentages of activation or inhibition in comparison to baseline were calculated every minute and correlated to the total cumulative dose perfused. The effect of the drug was evaluated by comparing the mean number of spikes $/ 10 \mathrm{~s}$ during the control period with the same value calculated during the last minute of perfusion (10th $\mathrm{min}$ ). When inhibition was observed, the mean total dose required to produce a $50 \%$ effect $\left(\right.$ ID $\left._{50}\right)$ was calculated by multiplying the dose perfused $\left(\mathrm{mg} \mathrm{kg}^{-1} \mathrm{~min}^{-1}\right)$ by the time (min) required to obtain $50 \%$ inhibition. In view of the rapid start of the drug effect, the lag between the beginning of the

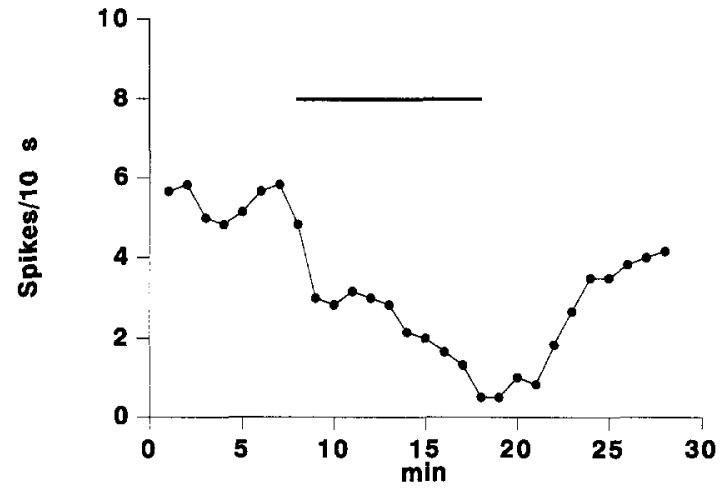

Fig. 1. The i.v. perfusion of JL3 induced a marked, dose-dependent and reversible decrease of the firing rate of this dorsal raphe neuron. The mean number of spikes $/ 10 \mathrm{~s}$ calculated every minute is represented against time. The bar indicates the period of perfusion.

perfusion and the beginning of the drug effect was not taken into consideration. Eight experiments were performed on every region studied. Only one cell was studied in each animal. JL3 was dissolved in saline slightly acidified with acetic acid ( $\mathrm{pH}$ 5.5-6.5). Statistical analysis of the results was performed using Student's $t$-test. Values are given as means \pm S.E.M.

\section{Results}

\subsection{Dorsal raphe neurons}

JL3 was perfused at the dose of $1 \mathrm{mg} \mathrm{kg}^{-1} \mathrm{~min}^{-1}$ for $10 \mathrm{~min}$. The drug induced a marked and significant $(P<$ 0.001 ) decrease of the firing rate of these serotonergic neurons. The effect was dose-dependent and reversible (Fig. 1). Fig. 2A summarizes the results obtained in 8 animals. The mean $\mathrm{ID}_{50}$ was $3.87 \pm 0.57 \mathrm{mg} \mathrm{kg}^{-1}$. Table 1 shows a comparison of the $\mathrm{ID}_{50}$ values obtained for JL3 and various antidepressant drugs previously studied under the same experimental conditions.

Table 1

Inhibitory potency of JL3 and various antidepressant drugs on the firing rate of dorsal raphe (DR) and locus coeruleus (LC) neurons

\begin{tabular}{lccll}
\hline & \multicolumn{4}{l}{$\mathrm{ID}_{50}\left(\mathrm{~kg}^{-1}\right)( \pm$ S.E.M. } \\
\cline { 2 - 5 } & DR & $n$ & LC & $n$ \\
\hline JL3 & $3.87 \pm 0.57$ & 8 & $2.63 \pm 0.35$ & 8 \\
Imipramine $^{\mathrm{a}}$ & $1.63 \pm 0.39$ & 6 & $1.30 \pm 0.11$ & 6 \\
Desipramine $^{\mathrm{a}}$ & $>12$ & 5 & $0.29 \pm 0.02$ & 6 \\
Clomipramine $^{\mathrm{a}}$ & $0.34 \pm 0.002$ & 6 & $3.08 \pm 0.3$ & 6 \\
Trazodone $^{\mathrm{b}}$ & $0.6 \pm 0.18$ & 6 & $>8$ & 6 \\
Fluvoxamine $^{\mathrm{b}}$ & $0.39 \pm 0.04$ & 6 & $>50$ & 6 \\
Fluoxetine $^{\mathrm{c}}$ & $2.2 \pm 0.29$ & 10 & $>10$ & 6 \\
\hline
\end{tabular}

$\mathrm{ID}_{50}=$ mean total dose required to produce a $50 \%$ inhibition of the frequency of discharge; $n=$ number of experiments.

${ }^{a}$ Data from Scuvée-Moreau and Dresse (1979); ${ }^{b}$ data from Dresse and Scuvée-Moreau (1984); ${ }^{c}$ unpublished data. 

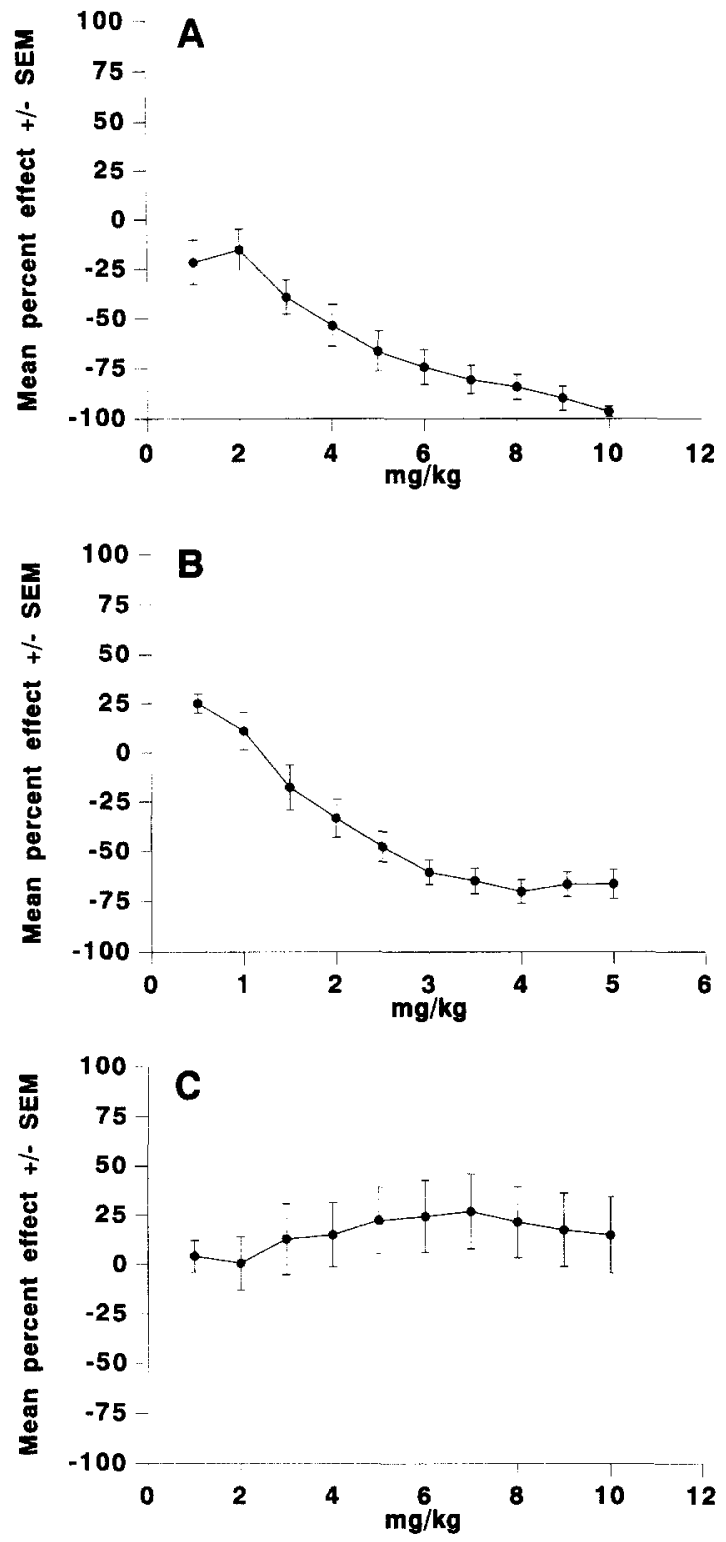

Fig. 2. Time course of the effect of i.v. perfusion of JL3 on the firing rate of (A) dorsal raphe serotonergic neurons, (B) locus coeruleus noradrenergic neurons, (C) A10 dopaminergic neurons. The mean percentages of activation or inhibition in comparison to baseline are presented in relation to the total cumulative dose perfused $(n=8)$.

\subsection{Locus coeruleus neurons}

$\mathrm{JL} 3$ was perfused at the dose of $0.5 \mathrm{mg} \mathrm{kg}^{-1} \mathrm{~min}^{-1}$ for 10 min. A marked and significant $(P<0.001)$ decrease of the firing rate of these noradrenergic neurons was observed. The effect was dose-dependent and reversible. Fig. 2B illustrates the results obtained in 8 animals. The mean $\mathrm{ID}_{50}$ was $2.63 \pm 0.35 \mathrm{mg} \mathrm{kg}^{-1}$. Table 1 shows a comparison of potency between JL3 and various antidepressant drugs. There was no significant difference between the $\mathrm{ID}_{50}$ values obtained for JL3 on dorsal raphe and locus coeruleus neurons $(P=0.08)$.

\subsection{Al0 neurons}

The i.v. perfusion of JL3, at the dose of $1 \mathrm{mg} \mathrm{kg}^{-1}$ $\min ^{-1}$, did not induce any significant modification of the firing rate of these dopaminergic neurons (Fig. 2C).

\section{Discussion}

These experiments clearly show the in vivo activity of JL3. Following acute i.v. administration, JL3 induced a marked decrease of the firing rate of both central serotonergic and noradrenergic neurons. JL3 acted in a similar dose range as other antidepressant drugs (Table 1). These electrophysiological observations are in agreement with our results previously obtained using psychopharmacological procedures (Bruhwyler et al., 1995).

In the open-field test in rats, JL3 significantly increased activity parameters (total ambulation and entry scores) and reduced the defecation score, reflecting an anxiolytic/disinhibitory potential as previously found with sulpiride and clozapine (Bruhwyler et al., 1990) or ritanserin (unpublished results). Furthermore, in the forced swimming test in mice (Porsolt's test), JL3 induced a dose-related decrease in the immobility time without having a stimulant effect. In this classical test used for the screening of new potential antidepressant compounds, JL3 appeared equipotent to imipramine (Bruhwyler et al., 1995).

Previous studies showed that tricyclic antidepressant drugs acutely decreased the firing rate of central monoaminergic neurons (Scuvée-Moreau and Dresse, 1979; Dresse and Scuvée-Moreau, 1984), an effect which appeared well correlated with their potency to block amine uptake in vitro (Quinaux et al., 1982). As concerns locus coeruleus neurons, presynaptic $\alpha_{2}$-adrenoceptors probably mediate the feedback inhibition seen after acute administration of tricyclic antidepressants (Svensson et al., 1981). However, somatodendritic 5-HT 1 autoreceptors could mediate the feedback inhibition of dorsal raphe neurons (Blier and De Montigny, 1994). The receptors involved in the mediation of the clinical effect of antidepressant drugs remain a matter of debate as do the neurobiological mechanisms underlying their delayed onset of action. JL3 apparently has no effect $\left(\mathrm{IC}_{50}>10^{-4} \mathrm{M}\right)$ on 5-hydroxytryptamine and noradrenaline re-uptake (unpublished results). Binding experiments revealed that JL3 has negligible affinity $\left(K_{\mathrm{i}}>1000 \mathrm{nM}\right)$ for dopamine $\mathrm{D}_{2}$ and $\mathrm{D}_{1}$ receptor sites (Liégeois et al., 1994), $\alpha_{1}$ - and $\alpha_{2}$-adrenoceptors or benzodiazepine receptors (unpublished results) while it possesses appreciable affinity for $5-\mathrm{HT}_{1 \mathrm{~A}}\left(K_{\mathrm{i}}=323 \mathrm{nM}\right.$ ) (unpublished results), $5-\mathrm{HT}_{2 \mathrm{~A}}\left(K_{\mathrm{i}}=282 \mathrm{nM}\right)$, and muscarinic acetylcholine $\left(K_{\mathrm{i}}=209 \mathrm{nM}\right)$ receptor sites (Liégeois et al., 1994). Among these binding affinities, the $5-\mathrm{HT}_{1 \mathrm{~A}}$ receptor affinity could probably explain the inhibitory effect of JL3 on the firing rate of dorsal raphe neurons. Furthermore, selective $5-\mathrm{HT}_{1 \mathrm{~A}}$ receptor agonists have been shown 
to have antidepressant properties in humans (Blier and De Montigny, 1994). However, a stimulation of $5-\mathrm{HT}_{2}$ receptors by JL3 could be involved in its inhibitory effect on locus coeruleus neurons. Indeed, it was previously shown that the spontaneous discharge of locus coeruleus neurons may be under serotonergic influence probably via postsynaptic 5- $\mathrm{HT}_{2}$ receptors (Gorea and Adrien, 1988).

Anyway, further work will be necessary to establish the precise mechanisms involved in the electrophysiological effects of JL3.

In conclusion, JL3 exhibits a profile of activity considered to be predictive of an antidepressant action. Although the neurochemical substrate for this action is still under investigation, it is tempting to speculate that the atypical antidepressant-like effect of JL3 on both noradrenergic and serotonergic neurons is related to its selective action on $5-\mathrm{HT}_{1 \mathrm{~A}}$ and $5-\mathrm{HT}_{2}$ receptors.

\section{Acknowledgements}

The authors gratefully acknowledge the Fonds National pour la Recherche Scientifique (FNRS Belgium) for the financial support. J.-F.L. is Senior Research Assistant of the FNRS Belgium.

\section{References}

Aghajanian, G.K., R.Y. Wang and J. Baraban, 1978, Serotonergic and non-serotonergic neurons of the dorsal raphe: reciprocal changes in firing induced by peripheral nerve stimulation, Brain Res. 153, 169.

Blier, P. and C. De Montigny, 1994, Current advances and trends in the treatment of depression, TIPS 15, 220.

Bruhwyler, J., E. Chleide, J.-F. Liégeois, J. Delarge and M. Mercier, 1990, Anxiolytic potential of sulpiride, clozapine and derivatives in the open-field test, Pharmacol. Biochem. Behav. 36, 57.
Bruhwyler, J., J.-F. Liégeois, E. Chleide, F. Rogister, J. Damas, J. Delarge and M. Mercier, 1992, Comparative study of typical neuroleptics, clozapine and new synthesized clozapine analogues: correlations between neurochemistry and behaviour, Behav. Pharmacol. 3, 567.

Bruhwyler, J., J.-F. Liégeois, C. Lejeune, F. Rogister, J. Delarge and J. Géczy, 1995, New dibenzoazepine derivatives with disinhibitory/ anxiolytic and/or antidepressant potential: behavioural study in the open-field and forced swimming tests, Behav. Pharmacol. 6, 830 .

Bunney, B.S., J.R. Walters, R.H. Roth and G.K. Aghajanian, 1973, Dopaminergic neurons: effect of antipsychotic drugs and amphetamine on single cell activity, J. Pharmacol. Exp. Ther. 185, 560.

Dresse, A. and J. Scuvée-Moreau, 1984, The effects of various antidepressants on the spontaneous firing rates of noradrenergic and serotonergic neurons, Clin. Neuropharmacol. 7, Suppl. 1, S313.

Gorea, E. and J. Adrien, 1988, Serotonergic regulation of noradrenergic coerulean neurons: electrophysiological evidence for the involvement of 5- $\mathrm{HT}_{2}$ receptors, Eur. J. Pharmacol. 154, 285.

Korf, J., B.S. Bunney and G.K. Aghajanian, 1974, Noradrenergic neurons: morphine inhibition of spontaneous activity, Eur. J. Pharmacol. $25,315$.

Liégeois, J.-F., J. Bruhwyler, J. Damas, T.P. Nguyen, E. Chleide, M. Mercier, F. Rogister and J. Delarge, 1993, New pyridobenzodiazepine derivatives as potential antipsychotics: synthesis and neurochemical studies, J. Med. Chem. 36, 2107.

Liégeois, J.-F., F. Rogister, J. Bruhwyler, J. Damas, T.P. Nguyen, M.O. Inarejos, E. Chleide, M. Mercier, F. Rogister and J. Delarge, 1994, Pyridobenzoxazepine and pyridobenzothiazepine derivatives as potent central nervous system agents: synthesis and neurochemical studies, $\mathrm{J}$. Med. Chem. 37, 519.

Quinaux, N., J. Scuvée-Moreau and A. Dresse, 1982, Inhibition of in vitro and ex vivo uptake of noradrenaline and 5-hydroxytryptamine by five antidepressants; correlation with reduction of spontaneous firing rate of central monoaminergic neurons, Naunyn-Schmiedeberg's Arch. Pharmacol. 319, 66.

Scuvée-Moreau, J. and A. Dresse, 1979, Effect of various antidepressant drugs on the spontaneous firing rate of locus coeruleus and dorsal raphe neurons of the rat, Eur. J. Pharmacol. 57, 219.

Svensson, T.H., C. Dalhöf, G. Engberg and H. Hallberg, 1981, Central pre- and postsynaptic monoamine receptors in antidepressant therapy, Acta Psychiat. Scand. Suppl. 290, 63, 67. 